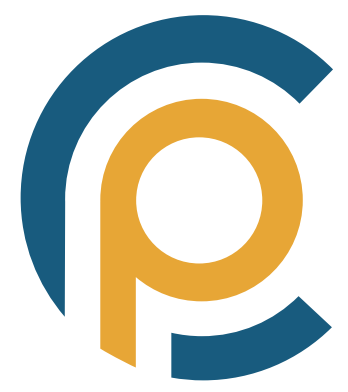

\title{
¡Cómo Hemos Cambiado! Medio Siglo de Escuela en España (1970-2020)
}

\author{
How We Have Changed! \\ Half a century of school in Spain (1970-2020)
}

Recibido: 07/10/2020 | Revisado: 04/11/2020 | Aceptado: 08/11/2020 | Publicado: 22/12/2020

Resumen: A las puertas de una nueva reforma educativa, conmemoramos la efeméride de la Ley General de Educación, un momento histórico que supuso una ruptura con la tradición escolar anterior. El objetivo de este trabajo es repasar alguno de los aspectos más notables de los cambios experimentados por la escuela en estos últimos cincuenta años.

Conscientes de la imposibilidad de agotar la temática, nos centramos en los fundamentos de cada uno de los cambios que llevan consigo los modelos de escuela y los diversos estilos pedagógicos que los sustentan.

Abordamos la "escuela de la racionalidad crítica" de los años 70 y 80 , propiciada por la Ley General de Educación (LGE), cimentada en los principios de la "pedagogía comprensiva" y la búsqueda del rendimiento educativo; la "escuela de la transversalidad" de los 90, bajo el enfoque de los modelos de enseñanza centrados en el aprendizaje del estudiante y la "pedagogía cognitiva del constructivismo" de la Ley de Ordenación General del Sistema Educativo (LOGSE); la "escuela de las competencias", a partir de la segunda mitad de la primera década de siglo, vinculada a teorías pedagógicas más alternativas y los enfoque de la Ley Orgánica de Educación (LOE): y, en un análisis orientado al futuro, la "escuela digital", que emerge por el horizonte con la inexcusable preeminencia de la virtualidad como medio pedagógico en los procesos de enseñanza-aprendizaje.

Palabras clave: escuela, enseñanza primaria, educación, aprendizaje, formación de docentes, profesorado.
Abstract: On the eve of a new educational reform, we are commemorating the anniversary of the General Law of Education, a historic moment that marked a break with the previous scholar tradition. The objective of this work is to review the changes that the school has experienced in the last fifty years. Being aware of the impossibility of draining the subject, we focus on the fundamental aspects of each change that school models bring about and the various teaching styles that support them.

We address the "school of critical rationality" of the 70 s and 80 s, based on the principles of "comprehensive pedagogy" and the search for educational performance, provided by the General Law of Education (LGE); the "school of transversality" of the 90s, under the approach of teaching models focused on student learning and the "cognitive pedagogy of constructivism" of the Law of General Organization of the Educational System (LOGSE); the "school of competences", from the second half of the first decade of the century, linked to more alternative pedagogical theories and the approaches of the Organic Law of Education (LOE): and, in a prospective analysis of the future, the "digital school" that emerges over the horizon, with the inexcusable preeminence of virtuality as a pedagogical resource in the teaching-learning processes.

Keywords: school, primary education, education, learning, teacher training, teachers. 
"Discúlpeme, no le había reconocido:

he cambiado mucho"

(O. Wilde)

\section{Introducción}

No resulta muy habitual, para algunos un tanto atrevido, iniciar un artículo con visos académicos evocando una canción de un grupo de música pop como "Presuntos Implicados"; la decisión se convierte en osadía, incluso, al destacarlo como parte integrante del título. No es una cuestión "friki" o de vano oportunismo, pese a lo que pueda parecer, sino de criterio didáctico en la concreción expositiva de la temática que se va a tratar, toda vez que refleja de manera precisa lo experimentado en esta diacronía histórica objeto de nuestro estudio: "Cómo hemos cambiado, qué lejos ha quedado". Y así es: no solo queda lejos, muy lejos, la Ley General de Educación (LGE), con medio siglo a sus espaldas, efeméride conmemorativa de este monográfico, sino que la institución escolar ha sufrido tantos cambios, que desde la mirada del presente nos sorprendemos al realizar este análisis retrospectivo.

Este, y no otro, es el objetivo que perseguimos a lo largo de estas páginas: resaltar alguno, y solo alguno, de los aspectos más notables de los cambios experimentados por los distintos modelos escolares en esta larga evolución histórica de la escuela española. Somos conscientes de la extensión de la temática y de las limitaciones espaciales y de contenido de un artículo de estas características, por lo que no espere el lector un análisis sesudo de investigación rigurosa sobre el amplio enfoque de dimensiones de la institución escolar en este medio siglo, ni un análisis pormenorizado de toda la legislación escolar. Centraremos nuestra atención en señalar los elementos del cambio más notables en tres momentos concretos: la drástica ruptura con el modelo de escuela anterior de la propia Ley General de Educación de 1970, su posterior reforma integral por la Ley Orgánica de Ordenación General del Sistema Educativo (LOGSE) en 1990 y la llegada de las competencias al mundo escolar por la Ley Orgánica de Educación de 2006 (LOE); no nos sustraemos, además, dada la situación especial en la que nos encontramos, a dedicar un último apartado, a modo de reflexión conclusiva, a la prospección del futuro, tan inmediato como incierto, y a las orientaciones que van a marcar el porvenir de la escuela. Para simplificar la búsqueda y perfilar la temática, utilizaremos tres parámetros descriptores que nos servirán de guía expositiva del contenido del trabajo y la significación de los cambios en cada momento histórico: "qué" se enseña, "cómo" se enseña y, lo que es más importante, el "para qué" de la enseñanza.

Hablamos, por tanto, de modelos de escuela (Gómez García, 2006) en sintonía directa con los enfoques pedagógicos que llevan implícitos (López Martín, 2005, 2019a) y que van jalonando el devenir histórico. La escuela ideológica de la posguerra vinculada a la "pedagogía suplantada" del primer franquismo, donde los valores de militancia patriótica y religión católica constituyen la única directriz orientadora, en una escuela de pobreza material y abundancia espiritual, representará un primer antecedente del que no vamos a ocuparnos en esta ocasión (López Martín, 2017); los inicios de la apertura socioeconómica de mitad de los años 50 propiciarán la escuela tecnocrática, donde la "pedagogía oficializada" buscará el refugio de lo ideológico en la eficacia y el rendimiento escolar, constituye la referencia inmediata de nuestro tema de análisis.

A partir de aquí, objetivos, enseñanzas mínimas, niveles de referencia, competencias, resultados de aprendizaje, áreas globales de contenido, unidades didácticas, fichas de individualización, medios audiovisuales, materiales didácticos, nuevas tecnologías... son conceptos que irán desfilando por el imaginario de la historia de la escuela en España durante estas últimas décadas y que suponen los ingredientes fundamentales de los modelos escolares a tratar en la época de estudio elegida: la escuela de la racionalidad crítica de los años 70 y 80 , sustentada en los principios de la "pedagogía comprensiva" y la búsqueda del rendimiento educativo; la escuela de la transversalidad de los 90 , bajo el enfoque de los modelos de enseñanza centrados en el aprendizaje del estudiante y la "pedagogía cognitiva del constructivismo"; la "escuela de las competencias", a partir de la segunda mitad de la primera década de siglo, vinculada a teorías pedagógicas más alternativas; y, finalmente, la escuela digital que emerge por 
el horizonte, con la inexcusable preeminencia de la virtualidad como recurso pedagógico en los procesos de enseñanza-aprendizaje.

\section{Una revolución silente y pacífica. La escuela de la racionalidad crítica}

Durante el régimen franquista, como decimos en la introducción, se había constatado el tránsito de una orientación pedagógica basada en la militancia patriótica y católica al reclamo de eficacia didáctica, de unas apologéticas llamadas a la "renacionalización" de la sociedad española hacia una labor escolar centrada en la "racionalización" de sus tareas, de una concepción de los maestros como "generales de la paz" a otra que los consideraba como cooperadores de una revolución silenciosa (López Martín, 2001, p. 150). Y eso era, entre otros aspectos, la nueva Ley General de Educación en los albores de la década de los años setenta del siglo pasado: una revolución pacífica y silenciosa, como la calificó su progenitor, que plantea la reforma del sistema educativo y su adaptación a las nuevas exigencias de racionalidad de la sociedad del tardofranquismo, con el anuncio, no sin cierto espíritu crítico, de un futuro de cambio que ya se percibía como algo ineludible.

Una de las primeras medidas de Villar Palasí como ministro de Educación y Ciencia fue encargar la redacción de un Libro Blanco, orientado a impulsar la reforma del sistema educativo (MEC, 1969). Las dos partes que conforman su redacción son fruto de los objetivos encomendados: de un lado, la más extensa, realizar un diagnóstico crítico -en muchas ocasiones duro con ciertos aspectos de la política educativa del pasado-; de otro, ofrecer unas propuestas de cambio, a modo de solución de la problemática descrita, agrupadas -según su elaboración final- en 22 bloques que van desde la propuesta de una nueva estructura general, pasando por la aplicación de los principios de educación permanente y de igualdad de oportunidades, una necesaria "evaluación del rendimiento educativo" o el estudio de las directrices a seguir en el "proceso de adaptación al nuevo marco político-educativo".

Bajo estas directrices, el 4 de agosto de 1970 (BOE, 6-VIII) se promulga la Ley General de Educación y Financiamiento de la Reforma Educativa como punto de partida "para una renovación profunda del Sistema Educativo Nacional", cuyo objetivo fundamental, tal como se lee en las Nuevas Orientaciones Pedagógicas ${ }^{1}$, publicadas con posterioridad para el desarrollo de la Ley, será "proporcionar más y mejor educación a todos los españoles a través de la expansión y democratización de la enseñanza, por un lado, y de la elevación y mejoramiento de su calidad y rendimiento educativo, por otro". Significar el carácter de servicio público de la educación, la obligatoriedad del Estado por hacer efectivo el principio de igualdad de oportunidades, la apuesta por una adecuación de contenidos y métodos, la educación personalizada, el esfuerzo constante en la experimentación e innovación, la construcción de un servicio continuado de orientación educativa, la reforma de la formación inicial del profesorado y su perfeccionamiento docente, o establecer unos métodos didácticos que sirvan para fomentar la actividad, originalidad y creatividad de los escolares, entre otros (Mayordomo, 1998), serán los ejes nucleares de la reforma.

La hasta entonces enseñanza primaria y parte del bachillerato elemental, queda configurada en el tramo de Educación General Básica (E.G.B.), nivel de ocho cursos de estudio -entre 6 y 13 años-, dividida en dos etapas -de 6 a 10 y de 11 a 13-, de carácter globalizador y formativo, la primera, y moderada diversificación de enseñanzas, la segunda. El art. $16^{\circ}$ de la Ley nos ofrece un primer referente del "para qué" del diseño curricular de la escuela:

En la E.G.B., la formación se orientará a la adquisición, desarrollo y utilización funcional de los hábitos y de las técnicas instrumentales de aprendizaje, al ejercicio de las capacidades de imaginación, observación y reflexión, a la adquisición de nociones y hábitos religioso-morales, el desarrollo de aptitudes para la convivencia y para vigorizar el sentido de pertenencia a la comunidad

1 OM 2-XII-1970, por la que se aprueban las Orientaciones Pedagógicas para la E.G.B. (Cfr. Educación General Básica. Nueva Orientación Pedagógica, Vida Escolar, 124-125, 1970-1971, 11). El carácter transitorio de la norma, queda zanjado por la OM. 6-VIII-1971, que prorroga y completa las Orientaciones Pedagógicas para la E.G.B. (Vida Escolar, 128-130, 1971) y la OM 27-VII-1973, por la que se aprueban las Orientaciones Pedagógicas para la Educación Preescolar. El entramado legislativo se cierra con la Resolución de la Dirección General de Educación Básica de 21-X-1977, sobre Instrucciones de coordinación entre los primeros cursos de E.G.B. con el nivel de Educación Preescolar. 
local, nacional e internacional, a la iniciación en la apreciación y expresión estética y artística y al desarrollo del sentido cívico-social y de la capacidad físico-deportiva (LGE, 1970, art. 16²).

La revolución no era solo la declaración de ciertos fines de la educación, impensables años atrás y de dudoso encaje en el modelo político del régimen, sino la democratización de la enseñanza y la ruptura con una estructura llena de reválidas y pruebas de nivel, que condenaba a las clases sociales más desfavorecidas al abandono del sistema educativo apenas cumplidos los 10 años de edad ${ }^{2}$. Se postula, por el contrario, la implementación de un curriculum común, obligatorio y gratuito para todos los españoles de 6 a 14 años, en una clara defensa de la igualdad de oportunidades y una apuesta decidida por el principio de comprensividad de la escuela europea de la época (De Puelles, 1992; Sevilla Merino, 1999).

Los contenidos se estructuran a través de grandes áreas de aprendizaje, evitando la fragmentación en asignaturas: "áreas de expresión" (lenguaje, matemáticas, plástica y dinámica) y "áreas de experiencia" (social y cultural, ciencias de la naturaleza y formación religiosa); sin duda, la tendencia a la concentración de contenidos de las "unidades didácticas", sigue presente en esta reforma (Rodríguez Diéguez, 1990). El "qué" se debe enseñar viene marcado a modo de objetivos operacionales de progresión creciente, facilitando así al profesor su evaluación, control y revisión de las distintas unidades; si en la primera etapa de la E.G.B. se subordina la información a la formación, globalizando las unidades en torno a "centros de interés", en la segunda, va alcanzando una mayor importancia la adquisición y sistematización de contenidos, alrededor de asignaturas propiamente dichas.

La opción curricular elegida por la Ley se caracteriza por la "eficacia, revisión de los contenidos, introducción de nuevos métodos y técnicas de enseñanza, evaluación del rendimiento, racionalización y técnica pedagógica" (Beltrán, 1992, p. 202). Su implementación escolar resulta tremendamente sencilla: formular unos objetivos (metas científicamente comprobables), instrumentalizar unos medios (dinámicos y modernos), desarrollar unas actividades coherentes con los fines propuestos, evaluar los resultados obtenidos y modificar el proceso en función de las desviaciones detectadas (De la Orden, 1968). Esta programación por objetivos, instaurada como nuevo modelo didáctico ${ }^{3}$, significa el punto de llegada de todo un planteamiento curricular iniciado en la década anterior, claramente orientado a la instrumentalización del curriculum; el proceso de enseñanza-aprendizaje queda reducido así a la concreción de los Cuestionarios en metas científicamente comprobables, diseñadas para cubrir unos objetivos (niveles de promoción) propuestos por una serie de "especialistas" nombrados por la administración educativa, por lo que al maestro se "le desposeía de su legitimidad tradicional para determinar el qué y el para qué, limitándose al cómo y, aun eso, en escasa medida" (Beltrán, 1991 p. 201). Sin duda, el hecho provocará la falta de complicidad de un profesorado que se siente suplantado en la gestión de una nueva escuela para la que no había sido formado, ni escuchado (Navarro Sandalinas, 1992). Esta desafección, sin duda, explica una buena parte del fracaso de la Reforma.

El trabajo práctico de la escuela (López Martín, 2002), atendiendo a la perspectiva metodológica del "cómo enseñar", se configura a través de las fichas de individualización de la enseñanza, cuya finalidad es "la realización de actividades, sugerencias y ejercicios a través de los cuales el alumno llega a adquirir determinados conocimientos y hábitos programados como objetivos" ${ }^{\text {. }}$. Sus funciones caminan por la fijación de conocimientos, proporcionando la práctica necesaria para reforzar el aprendizaje, la aplicación de éstos a experiencias relacionadas con las unidades programadas, y aun la posibilidad de ayudar a superar dificultades de aprendizaje. Además de estas "fichas de trabajo", nos encontramos con

2 Blat Gimeno (1992, p. 294), uno de los colaboradores más cercanos en la elaboración de la Reforma, años después, señalará como uno de los mayores logros de la Ley el conseguir romper la estructura tradicional de elitismo en los estudios posprimarios, al conseguir "la generalización del derecho a la educación y a la igualdad de oportunidades educativas".

3 Una breve y clarificadora exposición de esta formulación de la programación, en Medina Rubio (1970), director del Centro de Orientación y Documentación Didáctica de Enseñanza primaria (CEDODEP), organismo encargado de traducir los saberes expertos de la ciencia pedagógica a la realidad curricular de las escuelas españolas. Asimismo, Mª. A. Galino (1970), para entonces Directora General de Enseñanza Media y Profesional, ofrecerá una clara argumentación del sentido del concepto de programación escolar.

4 "Las fichas de trabajo", Vida Escolar, 145-46 (1973) 4-5. Un análisis didáctico más profundo de este recurso, puede consultarse en Martínez Sánchez (1998). 
"fichas-guía" que orientan al alumno en el proceso de trabajo o lo remite al manual de consulta, "fichas de información" que añaden contenido necesario para su trabajo, o "fichas de control" destinadas a facilitar la evaluación.

Este proceso de individualización de la enseñanza vendrá apoyado, entre otros factores, por la primera ola de modernización del utillaje escolar de la segunda mitad del siglo XX (López Martín, 2006). Junto a la persistencia de los objetos escolares tradicionales, asistimos en los años setenta al inicio de la generalización escolar de los medios audiovisuales como recurso educativo del trabajo de aula; retroproyectores, laboratorios de idiomas, programas de radiotelevisión educativa y otras tecnologías, pasarán a formar parte de la vida cotidiana de nuestros centros docentes, no solo como herramientas capaces de transformar la práctica, sino como recursos para fomentar en el profesorado la investigación y la participación en experiencias de innovación curricular ${ }^{5}$. El art. $18^{\circ}$ de la Ley, dedicado a los métodos, precisa con claridad su objetivo: "habrán de fomentar la originalidad y creatividad de los escolares, así como el desarrollo de aptitudes y hábitos de cooperación, mediante el trabajo en equipo de profesores y alumnos".

No obstante, el nuevo marco político de la Transición Democrática exigirá una nueva reconfiguración del trabajo escolar: los fines de la educación ("para qué" se enseña), la validez de los saberes (el "qué") y la crítica de los métodos (el "cómo"), deberán ser repensados y adaptados. La Orden Ministerial de 29 de noviembre de 1976 (BOE, 3-XII) establece nuevos contenidos de "formación cívico-social" en las orientaciones pedagógicas del área de social en la segunda etapa de EGB, bajo la denominación de "Educación para la convivencia". "El sistema educativo -se lee en la introducción- constituye una base imprescindible para el logro de una sociedad democrática"; valores como convivencia, tolerancia, solidaridad, participación, se presentan como líneas formativas básicas para el nuevo objetivo escolar de formación de la ciudadanía que, poco después, tomará carta de naturaleza en la Constitución de 1978.

Pero serán los contenidos, el "qué" enseñar, los que sufrirán una reforma global, a través de los Programas Renovados de la $E G B^{6}$. Dos líneas argumentales razonan la pertinencia de la renovación curricular: los cambios sociopolíticos acaecidos y el requerimiento de nuevas demandas sociales, junto al nuevo marco jurídico producto de la primera ley educativa de la Transición, la Ley Orgánica del Estatuto de Centros Escolares (LOECE), de 1980, conforman la primera; el envejecimiento de los contenidos y los índices elevados de fracaso escolar, se agrupan en la segunda, ésta de carácter eminentemente técnico ${ }^{7}$. Considerada como "una transición curricular en el seno de la transición política" (Beltrán, 1991 p. 215), nuestro análisis selectivo nos lleva a destacar tres aspectos centrales en la reforma programada: la nueva configuración de la EGB en tres ciclos, la jerarquización del diseño curricular con la presentación de unos "niveles básicos de referencia" más estructurados y programados y, finalmente, la incorporación de contenidos inexcusables para la formación del nuevo ciudadano. De opiniones pedagógicas encontradas, en el primer caso, e inexcusable implementación, en el tercero, será el desarrollo curricular el aspecto más determinante en la corta vigencia de la reforma.

5 A este respecto, cabe señalar el Programa EDINTE (Programa para el Ensayo y Difusión de Nuevas Técnicas Educativas), creado en 1977 por la Dirección General de Educación Básica. A partir de 1979 será transformado en los llamados Planes de Desarrollo de la Innovación Educativa. A título de ejemplo, puede verse La innovación educativa en la EGB. Técnicas para el aprendizaje autónomo de los alumnos (1982), Vida Escolar, 215, n. ${ }^{\circ}$ monográfico dedicado a las experiencias llevadas a cabo en el marco del II Plan de Desarrollo de la Innovación en Educación Básica.

6 Documento Base de Introducción a los Programas Renovados de la EGB (1980), Vida Escolar, 206, separata 15 pp. Las Enseñanzas Mínimas y los Niveles Básicos de Referencia, en RD 69/1981 de 9 de enero (BOE, 17-1) y OM de 17-I-1981 (BOE, 21-1), para el ciclo inicial de la EGB; RD 710/1982 de 12 de febrero (BOE, 15-IV) y OM 6 de mayo del mismo año (BOE, 14-V), para el ciclo medio; RD 3087/1982 de 12 de noviembre (BOE, 22-XI) y OM del 25 del mismo mes (BOE, 4-XII), por su parte, completarán la renovación de los programas para el ciclo superior. Entre la abundante documentación de la época dirigida a los maestros para su interpretación en el ciclo inicial, cabe citar Programas Renovados para Preescolar y Ciclo Inicial (1981), Escuela Española y Los Programas Renovados de la EGB. Análisis, críticas y alternativas (1981) ICE, Universidad Autónoma de Madrid. Asimismo, el número monográfico de Cuadernos de Pedagogía, 76 (1981) abordará la temática. Para el ciclo medio, los monográficos de Vida Escolar, 216-17 (1982), Cuadernos de Pedagogía, 93 (1982) y el suelto Programas Renovados para el Ciclo Medio (1982), Escuela Española.

7 Según datos oficiales el $30 \%$ de los escolares no termina con éxito la Primaria y no alcanza el título de Graduado Escolar al final de la EGB ("La Educación General Básica a examen. Resultados y rendimiento del sistema educativo", Boletín de la Inspección de Educación Básica del Estado, 4, 1981, 7-104). 
Concebida como un juego de "muñecas rusas" (Salinas, 1994), donde la de mayor tamaño -el curriculum oficial y prescriptivo- alberga al resto, hasta llegar al nivel mínimo de la planificación de aula, esta estructura curricular parte de los ciclos, respetando el concepto de áreas, con las diversas materias de cada una de ellas, que quedan divididas en bloques temáticos o agrupaciones de contenidos; éstos, a su vez, presentan varios temas de trabajo con especificación de objetivos que el alumno deberá conseguir al final de cada curso o ciclo, además de una serie de actividades orientadas a su consecución. Con ello, el docente tiene "programado" todo el proceso de enseñanza-aprendizaje, donde lo importante no era la reflexión sobre qué contenidos deben enseñarse, sino la formulación correcta de los objetivos operativos y su grado de coherencia con las actividades a desarrollar y los mecanismos propuestos para la evaluación. El maestro, ahora todavía más, queda de tal forma instrumentalizado que su principal función consiste, simplemente, en reflejar en la "programación en doble folio cuadriculado" el grado de adquisición de objetivos realizado por cada uno de sus estudiantes. Plantear una "nueva escuela" era algo más que este simple trabajo de remozamiento del curriculum de la década anterior.

Así pues, el imaginario escolar de finales de esos años 80, con una escuela que había abandonado definitivamente las enciclopedias para abrazar renovados materiales didácticos, que veía incrementar los alumnos en las aulas y la diversidad de éstos, donde ya se habla de participación, de democracia, de colaboración profesores y alumnos, con la presencia de Asociaciones de Padres y Madres formando parte de la comunidad educativa, que asiste a la primera floración de elementos tecnológicos en las aulas que hacían obsoletos los medios audiovisuales del pasado, con maestros escasos de innovación metodológica, faltos de formación, sin apenas reconocimiento social y, sobre todo, olvidados por las instancias de determinación curricular...converge en una radiografía escolar que daba por finalizada la década, con el convencimiento de que la reforma profunda de la escuela resultaba imprescindible y, quizás también, la puesta en marcha de un nuevo contexto legal para la totalidad del sistema educativo.

\section{La escuela de la transversalidad en los años 90}

La Ley Orgánica 1/1990, 3 de octubre, de Ordenación General del Sistema Educativo, traerá consigo el modelo escolar que hemos denominado "escuela de la transversalidad", bajo los parámetros constructivistas de los principios de la pedagogía cognitiva (Coll, 1989, 1991). La comprensividad, la atención a la diversidad, los enfoques de enseñanza centrados en el aprendizaje del estudiante, las teorías del alineamiento constructivista, el aprendizaje significativo, el desarrollo de capacidades y no de objetivos como medida de logro escolar y los temas transversales serán, ahora, el ropaje pedagógico de la nueva escuela (Bolívar y Rodríguez Diéguez, 2002).

Calificada como una ley ambiciosa, reconfigura de manera notable la estructura del sistema educativo, al extender la enseñanza obligatoria de 6 a 16 años, como un periodo formativo común, organizado de forma comprensiva e integradora ${ }^{8}$, que abarca tanto la educación primaria (6-12 años) ${ }^{9}$, como la educación secundaria obligatoria (ESO), en un nuevo nivel educativo (12-16 años), que viene a poner fin a la segregadora doble titulación del final de la EGB. Esta nueva etapa, la ESO, constituye el reto más importante de la reforma, no solo por la reestructuración de los centros docentes y la diferente adscrip-

8 Así lo define el Libro Blanco para la Reforma del Sistema Educativo (M.E.C., 1987): "Por escuela comprensiva se entiende una forma de enseñanza que ofrece a todos los alumnos de una determinada edad un fuerte núcleo de contenidos comunes dentro de una misma institución y una misma aula, y que evita de esta forma la separación de los alumnos en vías de formación diferente que puedan ser irreversibles. En ella se condensan las aspiraciones de la igualdad de oportunidades, cultura superior y común para todos los alumnos y apertura a la diversidad dentro de una misma escuela".

9 El art. $12^{\circ}$ establece: "La educación primaria comprenderá seis cursos académicos, desde los seis a los doce años de edad. La finalidad de este nivel educativo será proporcionar a todos los niños una educación común que haga posible la adquisición de los elementos básicos culturales, los aprendizajes relativos a la expresión oral, a la lectura, a la escritura y al cálculo aritmético, así como una progresiva autonomía de acción en su medio". 
ción del profesorado, que debería adaptarse a la nueva coyuntura ${ }^{10}$, sino por las funciones a cumplir: preparar para estudios superiores (bien FP, bien Bachillerato) y culminar la formación de aquellos que no continúan en el sistema educativo, lo que exige una flexibilidad de tiempos, ritmos y metodologías.

La propuesta curricular, "abierta y flexible", supone una importante revolución, tal como expresa Marchesi (2001), uno de sus ideólogos:

"Se establecieron distintos niveles de decisión en el diseño del currículo: nacional, autonómico y específico de cada escuela; se apoyaron los proyectos curriculares de escuela; se definieron los objetivos educativos en términos de la capacidad de los alumnos; se modificará el sistema de evaluación; se dará una especial importancia al aprendizaje de procedimientos y actitudes; se incluirán contenidos transversales vinculados a la educación en valores de los alumnos en todas las áreas curriculares. Junto con estas propuestas que configuran el diseño del currículo, se impulsarán iniciativas relacionadas con la formación de los profesores y la elaboración de materiales para facilitar el desarrollo del currículo (p. 60)".

Toda una batería de cambios para una renovada lógica escolar.

La defensa de la igualdad -a la que la Ley dedica todo un Título-, como una concreción de la comprensividad democrática en consonancia con la diversidad, constituye otra de sus señas de identidad y del "para qué" de una escolarización inclusiva (Marchesi, 1992, 2020). Con el objetivo de hacer efectivo el derecho de todos a la educación se emprenden toda una serie de acciones de carácter compensatorio, como la ampliación de la oferta de educación infantil, la adopción de programas de adaptación de las enseñanzas a las necesidades de los alumnos con dificultades, la integración en los centros públicos de los alumnos con necesidades educativas especiales (art. 36.3), todo ello aderezado de una potente política de becas y ayudas al estudio.

Respecto a la metodología, el "cómo" enseñar, cabe anotar la novedad de que todos los recursos didácticos deben estar en consonancia con las teorías del aprendizaje significativo centrado en la actividad y participación del estudiante. Actividades individuales, de pequeño grupo, con agrupamientos flexibles, planificados con reflexión por parte del profesorado, dirigidos a motivar y facilitar la experimentación del alumno, fomentando el aprendizaje autónomo y la estructuración de conocimientos de los estudiantes, sin renunciar al uso de modernas tecnologías audiovisuales, incluso con la incipiente emergencia de las digitales, vendrán a cambiar la fisonomía de nuestras escuelas.

Además de las áreas de aprendizaje, por otra parte muy similares a las de la LGE, más allá de la aportación de las Administraciones Autonómicas y de los propios centros, en virtud de la descentralización curricular la nueva reforma, se señalan una serie de "temas transversales", planteados como un conjunto de elementos culturales, de sensibilización de actitudes, que impregnan todos los contenidos y objetivos de las áreas o disciplinas del currículum, a modo de ejes aglutinadores de los procesos de enseñanza-aprendizaje (González Lucini, 1994). La educación moral y cívica, la educación para la paz, la educación para la igualdad de oportunidades entre los sexos, la educación ambiental, la educación sexual, la educación para la salud, la educación del consumidor y la educación vial, son los campos señalados por el diseño curricular, que deberán corresponder a la tarea de todo el profesorado y, por extensión, a la totalidad de la comunidad educativa.

La cuestión de la transversalidad (Bolívar, 1996; Yus, 1998) apunta hacia cuál debe ser la cultura relevante que merezca ser enseñada, lo que entronca directamente con el "qué" y, sobre todo, el "para qué" de la educación. ¿Es conveniente un curriculum de contenidos instrumentales orientado a la futura profesionalización o debemos hacer hincapié en los aspectos de formación de la personalidad y de la

10 Hay que recordar la necesaria readaptación del rol del docente y sus dificultades: de un profesor tradicional de Bachillerato, de cierto resabio elitista, acostumbrado a la función de transmitir contenidos vinculados a su materia (saber profesional), a un grupo escaso de estudiantes interesados en promocionar a las universidades, se pasaba a las exigencias del modelo de la comprensividad, con alumnos adolescentes muy diversos en el aula, tanto de nivel como de intereses, con la responsabilidad de añadir a los contenidos actitudes y valores en la más pura esencia del maestro-educador (Bolívar, 1996); por otra parte, la convivencia en un mismo centro de maestros con profesorado de secundaria no resultó nada sencilla, al menos, en principio. Y todo ello, sin apenas formación y huérfanos de incentivos. Resulta muy ilustrativo el breve, pero intenso, artículo de J. Mª. Esteve (1991). 
socialización cívica y moral del futuro ciudadano? La LOGSE apuesta por lo segundo, toda vez que en su desarrollo ${ }^{11}$, los temas transversales llegaron a identificarse con la educación en valores, en la medida que integra conceptos y contenidos actitudinales. Los materiales para la reforma editados por el Ministerio (MEC, 1992a, 1992b, 1993), enviados gratuitamente a los centros, conocidos por el profesorado como "cajas rojas", dedican un cuaderno a la explicación de cada uno de los temas transversales y su implementación práctica en el curriculum escolar: no se trata, por poner un ejemplo, de saber de salud, cuanto de aprender y practicar conductas saludables (Gavidia-Catalán, Aguilar y Carratalá, 2011). Este despligue curricular dará paso a la introducción de nuevos documentos de gestión pedagógica y organizativa que, si bien supondrán una nueva exigencia para el profesorado, con el tiempo lograrán convertirse en hojas de ruta para el funcionamiento de los centros; la falta de información y de formación al profesorado en este momento, no obstante, centrará su aplicación en su vertiente más burocrática y administrativa.

Así pues, como se ha dicho, la inserción curricular de los temas transversales en los Proyectos Educativos de Centro y en la realidad práctica de los procesos de enseñanza-aprendizaje no resultó nada fácil (Palos, 2000). Además de las limitaciones señaladas, abordar los temas transversales exigía la conformación de equipos docentes cohesionados, lo que supone desterrar la cultura de la individualidad muy arraigada en el profesorado de la época: tradicionalmente, como hemos escrito no hace mucho (López Martín, 2019b, p. 88), "la escuela había dado respuestas colectivas (iguales para todos) basadas en el trabajo individual de los docentes; ahora, por el contrario, debería hacerse justo al revés: ofrecer respuestas individualizadas desde el trabajo colectivo de equipos docentes". Qué lejos quedaba -vuelvo a la letra de la canción citada- la escuela de apenas dos décadas atrás.

Bien es verdad, que las críticas posteriores al cambio escolar han sido lacerantes, no solo por la ausencia de rigor en su implementación, en algunos casos por falta de recursos y circunstancias ajenas a la propia reforma (Díaz Alcaraz, 2000), sino por la creencia de que estos valores actitudinales defendidos por la Ley suponían un abandono de los contenidos tradicionales y una merma en el nivel de adquisición de conocimientos, lo que explica -según los críticos- los males de la educación y, especialmente, los elevados índices de fracaso escolar. Autores como Moreno Castillo (2016), Sánchez Tortosa (2018) o Luri (2020), por citar solo algunos, claman en contra de esa "nueva pedagogía", consolidada por la LOGSE ${ }^{12}$, cargada de valores y actitudes en favor de metodologías centradas en el estudiante, en aspectos lúdicos, en la actividad de los alumnos producto exclusivo de la motivación de sus profesores, alejada de la cultura del esfuerzo, de la disciplina, con merma de la autoridad del profesorado y con un vaciamiento de la instrucción, como función prioritaria de la escuela, que nos ha llevado a unos niveles de contenidos ínfimos en nuestros escolares. No faltarán propuestas similares en otros países europeos, caso de Francia y la "obstinada bancarrota de la escuela francesa" (Lurçat, 2004), o en el ámbito anglosajón (Christodoulou, 2014), por citar solo algunos.

Con independencia de las críticas y las limitaciones señaladas -algunas contrarrestadas, con posterioridad, por las directrices marcadas por la propia OCDE-, se puso de manifiesto que la escuela no puede desentenderse de la formación axiológica de una serie de valores y contenidos actitudinales propios y necesarios para el desempeño de la vida personal y social del ciudadano de las democracias del siglo XXI, llámense como se quiera, y se mantenga o no el término de transversalidad. Así, la Ley Orgánica de Educación (LOE) de 2006 y su desarrollo legislativo, pese a no recoger el concepto de forma explícita, apunta en su art. 1210:

11 RRDD 1006 y 1007/1991, de 14 de junio, por el que se establecen las Enseñanzas Mínimas correspondientes a Educación Primaria y a Educación Secundaria Obligatoria (BOE, 26-VI) y RRDD 1334-35/1991, de 6 de septiembre, por el que se establece el currículo de la Educación Primaria y la Educación Secundaria Obligatoria (BOE, 13-IX).

12 El proceso había comenzado con la "egebeización del Bachillerato elemental" que, para algunos, suponía una merma notable de contenidos y de nivel de conocimientos con respecto a los planes de estudio anteriores (Viñao, 1992, p. 50). En el próximo número de la Revista del Colegio de Pedagogos y Psicopedagogos de la Comunidad Valenciana, Crónica, 6 (2021) hemos comprometido un trabajo ("Me confieso pedagogo. Alegato en favor de la Nueva Pedagogía") en el que, huyendo de una defensa numantina, propia de gremialismos exacerbados en favor del colectivo al que uno pertenece, trataremos de poner en valor las fortalezas del discurso pedagógico en su reflexión y vinculación a la escuela, sin esconder las debilidades de ese relato durante los últimos años. 
El Proyecto educativo del centro recogerá los valores, los objetivos y las prioridades de actuación, incorporará la concreción de los currículos establecidos por la Administración educativa que corresponde fijar y aprobar al Claustro, así como el tratamiento transversal en las áreas, materias o módulos de la educación en valores y otras enseñanzas.

Asimismo, conviene recordar la creación de una asignatura específica como la Educación para la Ciudadanía y los Derechos Humanos, que venía a aglutinar los aprendizajes de carácter cívico y social en una materia curricular. La Ley Orgánica para la Mejora de la Calidad Educativa (LOMCE), de 2013, por su parte, años después, hará referencia a "elementos transversales" en el art. $10^{\circ}$ del nuevo Decreto de Currículo Básico para Educación Primaria 126/2014, mencionando los lenguajes verbal, audiovisual e informático, la educación cívica y constitucional, el emprendimiento, la educación de personas discapacitadas, la igualdad efectiva hombre-mujer, la prevención y solución de conflictos, los valores de libertad, igualdad, pluralismo, paz, democracia, respeto a derechos humanos, rechazo a la violencia, desarrollo sostenible, actividad física y dieta equilibrada y la seguridad vial, como parámetros aglutinadores del curriculum (Rosales, 2015).

\section{La escuela de las competencias}

La Ley Orgánica de Educación (LOE), de 2006, introduce, como novedad, el término de "competencias básicas" dentro de la definición del currículum español ${ }^{13}$. "Especial interés -se lee en la Exposición de Motivos- reviste la inclusión de las competencias básicas entre los componentes del currículo, por cuanto debe permitir caracterizar de manera precisa la formación que deben recibir los estudiantes". Con ello, el sistema educativo español se alinea con el resto de países europeos que, atendiendo a las demandas de los cambios socio-económicos y del mercado laboral, así como al objetivo de consolidar la construcción de una ciudadanía europea, exigen de la educación un cambio hacia el modelo de competencias (Tiana, 2009). La OCDE, a finales de la centuria pasada, puso en marcha el "Proyecto DeSeCo (Design and Selection of Competencies)", identificando un conjunto de competencias básicas como objetivo central de la educación, en torno al cual debería organizarse todo el diseño curricular de los aprendizajes de la enseñanza obligatoria (Rychen y Salganik, 2003). Este enfoque, definitivamente ratificado por la Recomendación del Parlamento Europeo y del Consejo del 18 de diciembre de 2006, sobre las competencias clave para el aprendizaje permanente (2006/962/CE) ${ }^{14}$, tiene como objetivos básicos seleccionar y organizar los aprendizajes escolares considerados como imprescindibles, es decir, lo que el docente debe enseñar y el alumno aprender para ejercer una ciudadanía activa, incorporarse a la vida laboral y ser capaz de desarrollar un aprendizaje permanente a lo largo de toda su vida, en el transcurso de su educación obligatoria.

"La educación -se lee en el preámbulo de la Ley- es el medio más adecuado para garantizar el ejercicio de la ciudadanía democrática, responsable, libre y crítica, que resulta indispensable para la constitución de las sociedades avanzadas, dinámicas y justas", por lo que los años de escolarización obligatoria deberán proporcionar a la juventud una educación completa, tanto en conocimientos como en "las competencias básicas que resulten necesarias en la sociedad actual, que les permitan desarrollar los valores que sustentan la práctica de la ciudadanía democrática, la vida en común y la cohesión social, que estimule en ellos y ellas el deseo de seguir aprendiendo y la capacidad de aprender por sí mismos". Un renovado "para qué" de la educación nos anuncia una remozada escuela.

13 Bien es cierto que, en el ámbito de la formación profesional, ya había una tendencia a diseñar el curriculum en términos de comportamientos observables relacionados con el buen desempeño profesional. La Ley Orgánica de Cualificaciones y Formación Profesional (2002), otorga a las competencias un papel central en la configuración de las diferentes titulaciones profesionales, así como en los diseños curriculares correspondientes. Entiende las competencias como "el conjunto de conocimientos y capacidades que permitan el ejercicio de la actividad profesional conforme a las exigencias de la producción y el empleo" (art. 7.3).

14 Puede consultarse el texto completo de la disposición normativa en https://bit.ly/3nGyPKT

Recientemente, por Recomendación del Parlamento Europeo y del Consejo de Europa de 18 de diciembre de 2018 sobre las competencias clave para el aprendizaje permanente ha quedado actualizada. https://bit.ly/3jFNG5H. Un análisis de esta última en Valle (2019).

Por otro lado, el marco general del Programa "Education \& Training 2010", puede consultarse en https://bit.ly/36RYUQX 
En el desarrollo normativo de la LOE, producto del debate llevado a cabo años atrás (MEC, 2004), es donde se aborda explícitamente el tema de las competencias, tanto en cuanto a su identificación como a su plasmación curricular. Las normas que regulan las enseñanzas mínimas ${ }^{15}$ definen las competencias básicas como el verdadero epicentro del curriculum de la educación básica, "salario mínimo cultural", en el decir de Bolívar (2008, p. 17). Las ocho competencias clave señaladas son: competencia en comunicación lingüística, matemática, en el conocimiento y la interacción con el mundo físico, tratamiento de la información y competencia digital, competencia social y ciudadana, cultural y artística, competencia para aprender a aprender y autonomía personal. El ministerio y las administraciones públicas autonómicas deberán compartir el desarrollo de este marco normativo y posibilitar que los centros y profesores elaboren los Proyectos Educativos de Centro y los programas de cada área o materia con autonomía pedagógica (Sarramona, 2004). Ese, al menos, era el principio teórico.

Este nuevo planteamiento supone una novedad en nuestra tradición curricular:

Hasta ahora -escribe Tiana (2011, p. 69)- los currículos establecían los objetivos, los contenidos, la metodología y los criterios de evaluación para cada área y materia, pero a partir de la LOE incluyen también unas orientaciones acerca del modo en que cada una de éstas contribuye al logro de aquellas competencias básicas.

Bajo esta directriz, aunque algunas competencias tuvieran una relación directa con una determinada materia, todo el trabajo curricular de áreas y materias, incluso de otras actividades extracurriculares, deberán estar al servicio de la consecución de la totalidad de las competencias. Una vez más, la apuesta orientada a la formación de equipos docentes y al trabajo cooperativo de los profesores, como recurso metodológico idóneo, con independencia de los nuevos materiales y tecnologías utilizadas, supone el sustrato básico del "cómo enseñar".

Quizás por ello, no faltan autores que han mostrado sus reticencias a la hora de evaluar las posibilidades de éxito de esta reforma curricular. "¿Están los sistemas educativos preparados para aceptar el declive del dominio de las disciplinas? -se pregunta Perrenoud (2009, p. 62)-; ¿están dispuestos a invertir masivamente en otra práctica de enseñanza-aprendizaje?" En realidad, como acertadamente escribe Rué (2008, p. 5), la cuestión no era otra que "la ingenuidad de suponer que se trata de un concepto unívoco (el ideario de la reforma) y que basta con enunciarlo para que se aplique y se obtengan los resultados deseados". Incluso, desde una perspectiva sustancialmente crítica, se ha llegado a plantear si este modelo de enseñanza escolar por competencias no era otra cosa que resucitar el viejo paradigma de la pedagogía por objetivos, de la segunda mitad del siglo XX, solo que con un lenguaje tecnocrático que los camufla (Escudero, 2008); por otro lado, en esta misma dirección, el título del libro coordinado por Gimeno Sacristán (2008), Educar por competencias, ¿qué hay de nuevo?, resulta algo más que sintomático.

Y es que no podemos olvidar que este modelo integra un triple discurso sobre el concepto mismo de competencia (Zabalza, 2012): conceptual, técnico y cultural. El primero, el conceptual, elemento polémico y que ha suscitado numerosas críticas, se estructura en torno a su legitimidad: si las competencias responden a una determinada ideología política, si constituyen una pérdida de contenidos y/o conocimientos, si es una simple estrategia para situar la educación al servicio del poder de ciertos grupos socioeconómicos, o más aún, si responde simplemente a una jerga vacía y, en cualquier caso, pasajera; otro discurso bien diferente es el técnico que, con independencia de la toma de postura sobre el primero, busca los argumentos pedagógicos necesarios para el cumplimiento de los principios de la reforma: hablamos de cómo incorporar las competencias al curriculum, cómo establecer las relaciones oportunas con el resto de elementos, cómo conseguir que los procesos de evaluación ayuden a su desarrollo, en definitiva, cómo situar la ciencia pedagógica al servicio de una mejora del aprendizaje de los estudiantes. Finalmente, con un carácter más difuso y mucho menos visible, encontramos un tercer discurso cultural

15 RD 1513/2006, 7 de diciembre, por el que se establecen las Enseñanzas Mínimas de la Educación Primaria y RD 1631/2006, 29 de diciembre, por el que se establecen las Enseñanzas Mínimas de la Educación Secundaria Obligatoria. Consultar el anexo I de cada uno de estas disposiciones legislativas. 
que hace referencia a las actitudes de compromiso de los miembros de la comunidad educativa para trabajar por el cambio ${ }^{16}$. "Y si esos son los debates en los que nos hemos estado moviendo durante todos estos años -escribe Zabalza (2012, p. 12)-, no hemos hecho sino reproducir la propia estructura interna de las competencias: conocimientos (discusión sobre el concepto y su legitimidad), prácticas (discusión de cómo ponerlas en práctica y cómo evaluarlas) y actitudes (mayor o menor disposición a vincularse a los cambios)".

Como siempre, una vez más, sin tiempo para desarrollar los principios de la reforma y evaluar de manera completa sus fortalezas y debilidades, asistimos a un nuevo giro de discurso con la aprobación de la Ley 8/2013, de 9 de diciembre, para la Mejora de la Calidad Educativa (LOMCE) y su nueva estructura curricular para la educación primaria por RD 126/2014, de 28 de febrero. Pese a mantener el modelo por competencias (ahora solo siete), la clara diferencia de enfoque ideológico nos llevará al predominio de los estándares de aprendizaje evaluables y los criterios de evaluación, junto a las pruebas finales de etapa ( $3^{\circ}$ y $6^{\circ}$ de Primaria), a modo de reválidas, con una estructuración curricular claramente diferente, en la que no vamos a entrar aquí.

\section{La preeminencia de lo digital. La escuela del futuro}

Si la preeminencia de lo digital sobre lo analógico, entre otras realidades emergentes, era ya una tendencia acusada en el ámbito educativo de estos últimos años, las consecuencias del cambio de paradigma provocado por la irrupción de la pandemia y la crisis sanitaria que estamos padeciendo fruto del Covid-19, con el obligado e inesperado cierre de las instituciones docentes, ha venido a acelerar la velocidad de ese cambio y a poner en valor la necesidad de volver a repensar los pilares de los procesos de aprendizaje: el qué, el cómo y el para qué en la educación escolar cobran una nueva dimensión (López Martín, 2020). Aprovechar la necesidad del cambio como oportunidad de mejora, hacer de la necesidad virtud, como suele decirse, deberá ser el relato pedagógico del futuro escolar de los próximos años.

Por ello, nos ha parecido oportuno cerrar este análisis evolutivo de algunos de los aspectos nucleares de los cambios experimentados por cada modelo escolar en este último medio siglo, más que con un apartado de conclusiones "al uso", con un apunte sobre las tendencias escolares actuales que, con mayor certeza constituirán las directrices del cambio del inmediato futuro. De otro lado, conscientes de que el futuro no surge de la nada, en el vacío, sino que se construye desde el presente, de tal forma que en el momento de imaginar los escenarios venideros contamos con una serie de indicios, de huellas, que nos permiten aventurar los parámetros argumentales del porvenir, utilizaremos estos comentarios finales como reflexión conclusiva de lo expuesto a lo largo de nuestro trabajo.

Si hace algunos años, con el cambio de rumbo introducido por la LOE de 2006, habíamos apostado por el modelo de enseñar por competencias, entendiendo que los conocimientos tenían un carácter necesariamente perecedero y no podíamos aspirar a ofrecer a nuestros estudiantes todos los contenidos necesarios para abordar cualquier contingencia, por lo que era preciso dotarles de habilidades y actitudes que facilitaran el aprendizaje a lo largo de la vida, la actualidad nos ha confirmado las fortalezas de dicho modelo, no exento de debilidades a mejorar. Consolidar competencias de liderazgo, de aprender a aprender y a desaprender, de innovación, creatividad, implicación en el trabajo colaborativo, de fomento del aprendizaje autónomo, de compromiso con la transformación social y apuesta por la equidad, de actualización del componente cívico de los valores transversales de la LOGSE, entre otras realidades, son habilidades y actitudes con las que deberemos equipar a los estudiantes. La escuela, nuestros centros docentes, con los equipos directivos a la vanguardia, en el marco de una reclamada autonomía pedagógica, deberán convertirse en talleres experienciales para el aprendizaje de estos valores y facilitar la capacidad de convertir las amenazas en oportunidades de mejora.

16 Parte de este compromiso está recogido en la política territorial que establece la LOE y que supone una financiación compartida entre la Administración Central y las Administraciones Regionales en materia educativa. Esta convergencia se refleja en el trabajo realizado por muchos docentes en el desarrollo de Programas de refuerzo, acompañamiento, orientación escolar (PROAs) que han puesto de manifiesto algunas de las claves del éxito educativo. 
Y en este reimaginar la vuelta a la ansiada normalidad una vez superada la emergencia sanitaria, hay otro aprendizaje que debe asumirse de manera definitiva, vinculado -en este caso- al cómo aprendemos: lo analógico y lo digital han quedado fusionados, cuando menos, en un solo mundo; no podemos renunciar al potencial de la virtualidad en el ámbito educativo y su integración ha dejado de ser una mera opción para convertirse en una realidad inexcusable. Si cambiamos la forma en que trabajamos, en que sentimos, en que vivimos, deberemos cambiar la manera en que aprendemos. No se trata de renunciar al contacto de aula, ni trasladar lo presencial al escenario on.line, escondiendo nuestras inercias docentes bajo la pátina de la modernidad tecnológica (Díez y Gajardo, 2020). Debemos repensar la totalidad de los diseños curriculares con códigos digitales: no basta, simplemente, con incorporar las herramientas tecnológicas a la docencia para consumir conocimiento, es necesario rediseñar la docencia con el auxilio del mundo virtual, de enriquecer el conocimiento y reconstruir las experiencias de aprendizaje: wikis, blogs, redes sociales, videos de los edutubers... van a formar parte de la escuela de mañana. Las competencias digitales, aun en una situación de normalidad presencial, van a consolidarse -sino lo habían hecho ya- como elementos básicos del escenario educativo (Trujillo, 2020). Y esto no significa una rendición a la máquina, sino apostar por hacer más tecnológico al humano (profesorado y estudiantes) y más humana la tecnología (metodologías innovadoras que acompañen a las plataformas virtuales).

Finalmente, aunque quizás debiera ser lo primero, habrá que repensar el "para qué" enseñamos, el propósito nuclear de la educación. Reforzar la idea de que la educación debe ser un instrumento al servicio de la convivencia democrática y la construcción de una ciudadanía provista de valores cívicos y de la capacitación necesaria para su libre ejercicio, debe constituirse en el eje axial de la tarea educativa, tal como se ha venido pergeñando en la realidad escolar de este medio siglo desde la propia LGE de 1970. No significa, para nada, la renuncia a la transmisión del saber o al esfuerzo del estudiante para asentar un sólido nivel de conocimientos, como han intentado hacer ver algunas teorías antipedagógicas, pero resulta una evidencia que deberemos concebir "la escuela que llega" (Pallarés, Chiva, López Martín y Cabero, 2018) como un recurso para la promoción de la solidaridad, la paz, la tolerancia, el diálogo, la resiliencia, la capacidad de empatía, la gestión emocional...en definitiva, de descubrir la necesidad del otro, de cultivar una nueva humanidad en un mundo incierto. Transmitir el saber y enseñar a nuestros escolares a pergeñar una futura profesión que les permita "ganarse la vida", seguirá siendo una función imprescindible en el porvenir inmediato de la escuela, pero educar para "entender la vida" en un mundo en permanente cambio, algo inexcusable.

Por ello, a las puertas de una nueva reforma educativa anunciada, con la perspectiva de que la confrontación política va a impedir -una vez más- el necesario Pacto de Estado por la Educación, es responsabilidad de la comunidad educativa, en general, y de los profesionales de la educación, en particular, poner en valor aquellos aspectos que nos parecen ineludibles para afrontar con garantías el futuro de nuestras escuelas (Feito, 2020, Fundación Santillana, 2020). Además de las ideas esbozadas en este último epígrafe, sea cual sea el devenir político y parlamentario de la nueva reforma, el flexibilizar el curriculum, realizar una selección más adecuada de los contenidos, huir de la fragmentación disciplinar hacia una acusada puesta en valor de la interdisciplinariedad y transdisciplinariedad, reforzar la presencia de los valores cívicos como centro de una sociedad democrática, respetar la autonomía de los centros y fomentar la participación efectiva de los docentes, incorporar metodologías participativas que superen el individualismo docente y facilite la cooperación en los procesos de aprendizaje, repensar la formación inicial y permanente del profesorado, evitar la desprofesionalización con la rígida fijación de estándares, en ocasiones descontextualizados, impulsar la puesta en valor de los docentes y la tarea educativa como un reto de primer orden para la sociedad en general, en definitiva, apostar por una escuela que afiance las bases del aprendizaje a lo largo de la vida ofreciendo competencias a los estudiantes para aprender a aprender, a desaprender y a reemprender.

¡Cómo hemos cambiado, qué lejos ha quedado! 
Referencias

Beltrán Llavador, F. (1991). Política y Reformas Curriculares. Universitat de València.

Beltrán Llavador, F. (1992). La reforma del currículo. Revista de Educación, nº extraordinario sobre la Ley General de Educación, pp. 193-209. https://bit.ly/2SI8rBM

Blat Gimeno, J. (1992). Apuntes sobre la elaboración y aplicación de la reforma educativa de 1968-1970, Revista de Educación, nº extraordinario sobre la Ley General de Educación, 289-296. https://bit. ly/34TbqNI

Bolívar, A. (1996). Límites y problemas de la transversalidad. Revista de Educación, 309, 23-65. https:// bit.ly/34KEzu9

Bolívar, A. (2008). Competencias básicas y ciudadanía. Caleidoscopio 1, 4-32. https://bit.ly/39gUOhH

Bolívar, A. y Rodríguez Diéguez, J. L. (2002). Reformas y retóricas: la reforma educativa de la LOGSE, Ediciones Aljibe.

Coll Salvador, C. (1989). Concepción constructivista y planteamiento curricular. Cuadernos de Pedagogía, 168, 8-14.

Coll Salvador, C. (1991). Diseño Curricular Base y Proyectos Curriculares. Cuadernos de Pedagogía, 188, 8-14.

Christodoulou, D. (2014). Seven myths about education. Routledge.

De la Orden, A. (1968). Programas, niveles y trabajo escolar, en CEDODEP. Niveles, Cuestionarios y Programas escolares (pp. 123-127). MEC.

De Puelles Benítez, M. (1992). Tecnocracia y política en la Reforma Educativa de 1970. Revista de Educación, no extraordinario sobre la Ley General de Educación, 13-29. https://bit.ly/3luxHrA

Díaz Alcaraz, F. (2000). Evaluación del grado de aplicación de la LOGSE. Ensayos: Revista de la Facultad de Educación de Albacete, 15, 143-162. https://bit.ly/33Oke7U

Diez-Gutiérrez, E. y Gajardo-Espinoza, K. (2020). Educar y Evaluar en Tiempos de Coronavirus: la Situación en España. Multidisciplinary Journal of Educational Research, 10(2), 102-134. https://bit. ly/3iRA5H4

Escudero Muñoz, J.M. (2008). Las competencias profesionales y la formación universitaria: posibilidades y riesgos, Revista de Docencia Universitaria, 6 (2), 1-20. https://bit.ly/3iWH2He

Esteve, J. M. (1991). Los profesores ante la reforma. Cuadernos de Pedagogía, 190, 54-58.

Feito Alonso, R. (2020). ¿Qué hace una escuela como tu en un sitio como este? Catarata.

Fundación Santillana (2020). La escuela que viene. Reflexión para la acción. Fundación Santillana. https://bit.ly/3nzfOd0

Galino, Ma. A. (1970). Concepto actual de programación. Revista de Educación, 207-208, 7-13. https:// bit.ly/3719SE1

Gavidia-Catalán, V.; Aguilar Moya, R. y Carratalá Beguer, A. (2011).¿Desaparecen las transversales con la aparición de las competencias?. Didáctica de las Ciencias Experimentales y Sociales, 25, 131-148. https://bit.ly/2GZWoNS

Gimeno Sacristán, J. (Comp.) (2008). Introducción, en Educar por competencias, ¿qué hay de nuevo? (pp. 9-14). Morata.

Gómez García, Ma . N. (2006). La infancia escolarizada: un proceso contradictorio en A. Escolano Benito (Dir.), Historia Ilustrada de la Escuela en España. Dos siglos de perspectiva histórica (pp. 331-50), Fundación Germán Sánchez Ruipérez.

González Lucini, F. (1994). Temas transversales y educación en valores. Anaya.

López Martín, R. (2001). La escuela por dentro. Perspectivas de la cultura escolar en la España del siglo $X X$. Servicio de Publicaciones Universitat de València. 
López Martín, R. (2002). Una escuela de todos y para todos. Las prácticas escolares en la Transición Democrática. Historia de la Educación, 21, 67-80. https://bit.ly/3jTEdrl

López Martín, R. (2005). Práctica escolar y Renovación pedagógica. Cambios y resistencias en la cultura escolar de la España franquista. En E. Candeias Martín (Coord.). V Encontro Ibérico de História da Educaçao. Renovaçao Pedagógica (pp. 221-266), Alma Azul,. https://bit.ly/36WVIUq

López Martín, R. (2006). El utillaje escolar en la segunda mitad del siglo XX". En A Escolano Benito (Dir.), Historia Ilustrada de la Escuela en España. Dos siglos de perspectiva histórica (pp. 425-448), Fundación Germán Sánchez Ruipérez.

López Martín, R. (2017). El maestro y la pedagogía suplantada del franquismo de la posguerra. Apuntes desde la literatura. e-CO, Revista digital de Educación y Formación del Profesorado, 14,1-21. https://bit.ly/3lB30kv

López Martín, R. (2019a). La figura del maestro y la cultura pedagógica en la España franquista. Reflejos desde la literatura. Utopía y Praxis Latinoamericana, 87 (24), 97-112. https://bit.ly/2SNHBIt

López Martín, R. (2019b). La innovación docente en la formación del profesorado. Principios y directrices de futuro. Crónica, Revista Científico Profesional de la Pedagogía y Psicopedagogía, 4, 83-94. https://bit.ly/3iPsgSt

López Martín, R. (2020). Reflexiones educativas pos-Covid19. Recordando el futuro. Revista Internacional de Educación y Justicia Social (RIEJS), 9 (3) 127-140. https://bit.ly/3IB3EhV

Lurçat, L. (2004). La destruction de l'enseignement élémentaire et ses penseurs. François Xavier de Guibert.

Luri Medrano, G. (2020). La escuela no es un parque de atracciones. Una defensa del conocimiento poderoso. Ariel.

Marchesi, A. (1992). Educational Reform in Spain. International Review of Education, 38 (6), 591-607. https://bit.ly/30ZgZce

Marchesi, A. (2001). Presente y futuro de la Reforma Educativa en España. Revista Iberoamericana de Educación, 27, 57-76. https://bit.ly/2GXmroy

Marchesi, A. (2020). La LOGSE en la educación española. Breve relato de un cambio histórico. Avances en Supervisión Educativa. Revista de la Asociación de Inspectores de Educación en España, 33, 1-34. https://bit.ly/3iJKWmA

Martínez Sánchez, A. (2006). Materiales autoinstructivos. Fichas de enseñanza y libros programados en A. Escolano Benito, A. (Dir.). Historia llustrada del libro escolar en España. De la posguerra a la reforma educativa pp. 327-350, Fundación Germán Sánchez Ruipérez.

Mayordomo, A. (1998). Société et politique éducative dans l'Espagne franquiste. Histoire de l'Education, 78, 199-227. https://bit.ly/2Im2okz

MEC (1969). La educación en España. Bases para una política educativa. Secretaría General Técnica. Ministerio de Educación y Ciencia.

MEC (1989). Libro Blanco para la Reforma del Sistema Educativo. Centro de Publicaciones del Ministerio de Educación y Ciencia. https://bit.ly/30X6C8z

MEC (1992a). Materiales Curriculares Transversales de Educación Primaria. Ministerio de Educación y Ciencia. https://bit.ly/36Tf3Wf

MEC (1992b). Materiales Curriculares Transversales de Educación Secundaria Obligatoria. Ministerio de Educación y Ciencia. https://bit.ly/3dh0sFe

MEC (1993). Temas Transversales y Desarrollo Curricular. Ministerio de Educación y Ciencia.

MEC (2004). Una educación de calidad para todos y entre todos. Propuestas para el debate. Ministerio de Educación y Ciencia. https://bit.ly/2GFXb6R 
Medina Rubio, R. (1970). Aspectos fundamentales de los planes y programas de estudio en la Educación General Básica. Revista de Educación, 207-208, 28-33. https://bit.ly/32JCBu7

Moreno Castillo, R. (2016). La conjura de los ignorantes. De cómo los pedagogos han destruido la enseñanza. Editorial Pasos Perdidos SL.

Navarro Sandalinas, R. (1992). La Ley Villar y la Reforma del Profesorado. Revista de Educación, $\mathrm{n}^{\circ}$ extraordinario sobre la Ley General de Educación, 209-236. https://bit.ly/3jV896E

Palos, J. (2000). Estrategias para el desarrollo de los temas transversales del currículo. ICE-Horsori.

Pallarés, M.; Chiva, O.; López Martín, R. y Cabero, I. (2018) La escuela que llega. Tendencias y nuevos enfoques metodológicos. Octaedro.

Perrenoud, Ph. (2009). Enfoque por competencias. ¿Una respuesta al fracaso escolar?”. Revista Interuniversitaria de Pedagogía Social, 16,45-64. https://bit.ly/2IdErf6

Rodríguez Diéguez, J.L. (1990). Balance de urgencia de cuatro reformas curriculares. Bordón, 42 (3), 257-66.

Rosales López, C. (2015). Evolución y desarrollo actual de los Temas Transversales: posibilidades y límites. Foro de Educación, 13 (18), 143-160. https://bit.ly/2GTBPm0

Rubio Medina, R. (1970). La programación de estudios en el nivel de Educación General Básica. Vida Escolar, 124-125, 2-8.

Rué, J. (2008). Formar en competencias en la universidad: entre la relevancia y la banalidad. Revista de Docencia Universitaria, 6 (1) 1-19. https://bit.ly/372vCiu

Rychen, D.S. y Salganik, L.H. (2003). Key competences for successful and a well-functioning society. Hogrefe \& Huber Publichers. (Trad. cast.: Las competencias claves para el bienestar personal, económico y social. Málaga. Ediciones Aljibe, 2006).

Salinas Fernández, B. (1994). La planificación de la enseñanza: ¿técnica, sentido común o saber profesional? En J.F. Angulo y N. Blanco (Coords.). Teoría y desarrollo del curriculum (pp. 135-160), Ediciones Aljibe.

Sánchez Tortosa, J. (2018). El culto pedagógico. Crítica al populismo educativo. Akal Ediciones.

Sarramona, J. (2004). Las competencias básicas en la Educación Secundaria Obligatoria. CEAC.

Sevilla Merino, D. (1999). La escuela comprensiva en España (1970-1990), en J. Ruiz., A. Bernat, M. R. Domínguez, y V.M. Juan Borroy. (Eds.). La Educación en España a examen (1898-1998) (pp. 265-277). MEC, Instituto «Fernando El Católico», vol. I. (CSIC).

Tiana Ferrer, A. (2009). Por qué hicimos la Ley Orgánica de Educación. Wolters Kluwer.

Tiana Ferrer, A. (2011). Análisis de las competencias básicas como núcleo curricular en la educación obligatoria española. Bordón. Revista de Pedagogía, 63 (1), 63-75, https://bit.ly/3iUBmgx

Trujillo, F. (2020). Aprender y enseñar en tiempos de confinamiento. Catarata.

Valle, J.M. (2019). Algunas luces y muchas sombras en la implantación de las políticas educativas supranacionales: el caso de las competencias clave en España. En A. De la Herrán Gascón, J.M. Valle López y J.L. Villena Higueras (Coords.). ¿Qué estamos haciendo mal en educación? Reflexiones pedagógicas para la investigación, la enseñanza y la formación (pp. 13-38). Octaedro.

Viñao Frago, A. (1992). La Educación General Básica entre la realidad y el mito. Revista de Educación, no extraordinario sobre la Ley General de Educación, 47-71. https://bit.ly/33Pwh59

Viñao Frago, A. (2020). La Ley General de Educación de 1970: ¿final de una etapa? ¿comienzo de otra? En M. Ferraz Lorenzo (Ed.). Modernización educativa y socialización política. Contenidos curriculares y manuales escolares en España durante el tardofranquismo y la transición democrática (pp. 125-150). Universidad de La Laguna, Editorial Morata. https://bit.ly/36UYIWD 
Yus, R (1998). Temas transversales. Hacia una nueva escuela. Graó.

Zabalza, M, A. (2012). La universidad de las competencias. Revista de Docencia Universitaria, 10 (2) 11-14. https://bit.ly/2JNDCuh 